\title{
Valproate reduces alcohol consumption in people with comorbid alcohol dependency and bipolar disorder
}

Salloum IM, Cornelius JR, Daley DC, et al. Efficacy of valproate maintenance in patients with bipolar disorder and alcoholisma double-blind placebo-controlled study. Arch Gen Psychiatry 2005;62:37-45.

\section{$Q$ Is valproate an effective adjuvant treatment for bipolar disorder with co-occurring alcohol dependence?}

\section{METHODS}

$\square$

Design: Randomised controlled trial.

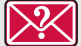

Allocation: Not reported.

Blinding: Double blind (assessors and participants blinded to treatment group)

Follow up period: Twenty four weeks.

Setting: University of Pittsburgh Medical Center, USA.

Patients: Fifty nine men and women aged 18-65 years with both an alcohol use disorder (meeting four of seven DSM-IV criteria for alcohol dependence) and an acute episode of bipolar I disorder. Exclusion criteria included: schizophrenia or schizoaffective disorder, non-bipolar psychotic disorder, opioid or cocaine dependence or current use of intravenous drugs, epilepsy or history of organic brain syndrome or brain injury, unstable medical conditions, and elevated liver enzymes. Women were excluded if they were pregnant or breast feeding.

$\mathbf{R}_{\mathbf{X}}$

Intervention: The intervention consisted of valproate (divalproex sodium) at a starting dose of $750 \mathrm{mg} /$ day, increased until serum concentration reached 50 to $100 \mu \mathrm{g} / \mathrm{ml}$. The placebo group received identical capsules twice daily. All participants continued to receive usual care, consisting of lithium treatment and weekly individual psychosocial counselling, throughout the study.

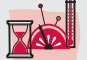

Outcomes: Proportion of heavy drinking ( $\geqslant 4$ drinks/day for women or $\geqslant 5$ drinks/day for men) days and number of drinks per heavy drinking day (measured using Timeline Follow-Back for Recent Drinking), remission of mania (measured using the BechRafaelsen Mania Scale (BRMS)), and remission of depression (measured using the Hamilton Rating Scale for Depression (HRSD-25)), assessed every four weeks.

Patient follow up: $20 / 59(38 \%)$ completed the study at 24 weeks.

\section{MAIN RESULTS}

Following treatment with valproate, there was a significant decrease in number of heavy drinking days and a non-significant reduction in drinks per heavy drinking day (people reporting heavy drinking days: $12 / 27(44 \%)$ with valproate $v 17 / 25(68 \%)$ with placebo; $\mathrm{p}=0.02$, number of drinks per heavy drinking days: 5.6 with valproate $v 10.2$ with placebo; $\mathrm{p}=0.055$ ), which became significant when adherence

For correspondence: Ihsan $M$ Salloum, $M{ }_{M D}, M P H$, Western Psychiatric Institute and Clinic of the University of Pittsburgh Medical Centre, 3811 O'Hara St, Pittsburgh, PA 15213, USA; salloumim@upmc.edu

Sources of funding: supported by a grant from the National Institute of Alcohol Abuse and Alcoholism (NIAAA). to treatment was controlled for in analysis $(p=0.02)$. Valproate also increased time to relapse of heavy drinking (days to relapse: 93 with valproate $v 62$ with placebo; $\mathrm{p}=0.048)$. Valproate had no effect on overall symptoms of mania or depression (BRMS score after treatment: 5.6 with valproate $v 6.1$ with placebo; $\mathrm{p}=0.87$, HRSD25 score after treatment: 16.3 with valproate $v 14.4$ with placebo; $\mathrm{p}=0.36)$, although there was a trend towards a reduction in time to remission of mania with valproate $(p=0.07)$. Mania and depression were strongly associated with alcohol consumption $(p=0.006)$.

\section{CONCLUSIONS}

In people with comorbid bipolar and alcohol abuse disorder, treatment with valproate is an effective adjunct to lithium and psychosocial counseling and reduces alcohol consumption. Valproate has little effect on mania and depressive symptoms.

\section{NOTES}

There was a very high dropout of participants in this study, so clinical significance of these results remain unclear. Participants were taking lithium and were having weekly psychosocial counselling throughout the study period.

\section{Commentary}

7 his seminal study will give hope to clinicians providing treatment to one of the most challenging populations in clinical practice-the alcoholic with co-occurring bipolar I disorder. Epidemiological estimates show that over half of those with a bipolar disorder diagnosis have an alcohol or drug use disorder. This population group is in dire need of a novel approach to stabilisation and improved quality of life, and the field has longed for an investigation on the use of a promising medication to reduce alcohol use outcomes. This is the first such study to show that valproate is clinically useful for decreasing heavy drinking in bipolar alcoholic patients.

When combined with bipolar disorder, alcohol dependence presents clinical challenges associated with heightened risk of suicide, poor medication compliance, multiple hospitalisations, and significantly increased medical complications. Poor treatment response is the rule rather than the exception, with severe social, family, and vocational dysfunction hindering treatment participation and progress. Now there is some clinical evidence of the efficacy of valproate in reducing alcohol use. By controlling heavy alcohol use the prognosis for treatment outcomes across several domains can be improved-such as decreased suicide risk, greater appointment compliance, and reduced medical complications.

It is essential that mental health and substance abuse care systems are enabled to deliver new pharmacotherapies and methods of care when they are shown to be effective. Individual practitioners must remain vigilant (and optimistic) that the efforts of clinical researchers can and will result in practical application to improve the lives of everyone in our communities.

Charlene E Le Fauve, PhD, Chief, Co-Occurring and Homeless Activities Branch, Substance Abuse and Mental Health Services Administration, Center for Substance Abuse Treatment, Rockville, MD, USA 